\title{
Use of Patterns in Formal Development: Systematic Transition From Problems to Architectural Designs
}

\author{
Christine Choppy ${ }^{1}$ and Maritta Heisel $^{2}$ \\ ${ }^{1}$ LIPN, Institut Galilée - Université Paris XIII, France, email: \\ Christine.Choppy@lipn.univ-paris13.fr \\ 2 Otto-von-Guericke-Universität Magdeburg, Fakultät für Informatik, Institut für Verteilte \\ Systeme,D-39016 Magdeburg, Germany, email: heisel@cs.uni-magdeburg.de
}

\begin{abstract}
We present a pattern-based software lifecycle and a method that supports the systematic execution of that lifecycle. First, problem frames are used to develop a formal specification of the problem to be solved. In a second phase, architectural styles are used to construct an architectural specification of the software system to be developed. That specification forms the basis for fine-grained design and implementation.
\end{abstract}

\section{Elaborating the software development process}

Experience has shown that problems and bugs in software systems take their source mainly in the early phases of the software development process ${ }^{1}$. Hence, a software development lifecycle that derives the design of the software directly from the requirements and then passes on to the implementation cannot be regarded as satisfactory. The step between requirements and design is too large.

An additional phase should be introduced between the requirements and the design. One idea that has been accepted for some time now is that some kind of specification should be set up on the basis of the requirements, so that the requirements are transformed into documents useful for developers. Specifi cations lead to a deeper understanding of the problems to be solved, and they can be used to support other development activities (e.g. coding, testing, maintenance). However, producing appropriate specifi cations often turns out to be diffi cult for practitioners. For instance, fi nding an appropriate starting point for the formal specifi cation process is a very common problem.

M. Jackson [Jac95,Jac01] proposes the use of problem frames for presenting and understanding software development problems. A problem frame is a characterization of a class of problems in terms of their main components and the connections between these components. A set of typical solution methods is associated to each problem frame. The basic idea is that once an appropriate problem frame for a given problem is found, we also have good proposals for constructing a solution to that problem. We think this idea

\footnotetext{
${ }^{1}$ See for example http://www.standishgroup.com/sample_research
} 


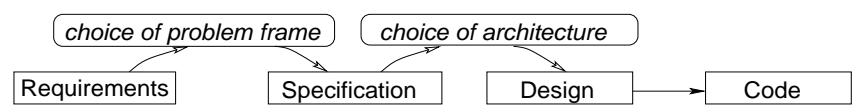

Fig. 1. Lifecycle using problem frames and architectures

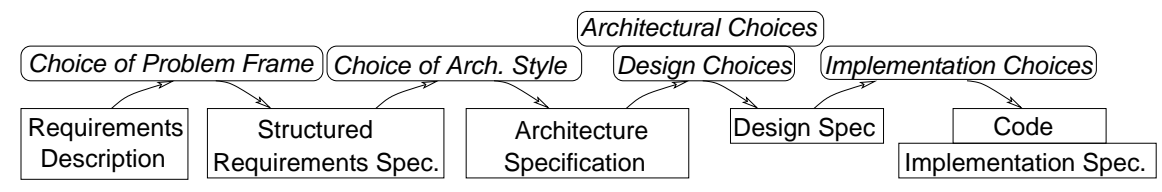

Fig. 2. Complete lifecycle using problem frames and architectural styles

is useful, but it provides only a coarse structure of the problem. Hence, problem frames should be supplemented by means that allow for a fi ner structuring.

Architectural styles [SG96,BCK98] are a means to structure a software system, i.e. to choose its architecture. Since architectural styles are used to construct designs, they should not be used right at the beginning of the development process, but only after the problem has been fully understood and specifi ed. Figure 1 shows how to bridge the gap between the requirements and the design of a software system. It is possible to elaborate the software development lifecyle further, as suggested in Figure 2. Here, several phases are introduced between the requirements and the design of a software system.

Problem frames and architectural styles are both forms of patterns. While problem frames are concerned with problems, architectural styles are concerned with solutions. Hence, with Figures 1 and 2, we propose a pattern-based software lifecycle. Patterns should be used systematically and on different levels of abstraction.

In the following, we show how the steps from an informal requirements description to an architectural specifi cation shown in Figure 2 can be carried out in a systematic way. This work further elaborates the approach by Choppy and Reggio [CR00], where problem frames are used to structure formal specifi cations.

We fi rst discuss how patterns can be used on different abstraction levels and in different phases of the software development process in Section 2. Section 3 presents a method to carry out pattern based formal development in a systematic way. The application of that method is illustrated by the case study of a robot simulation in Section 4. In Section 5, we summarize our work and also discuss related work that aims at methodological support for developing formal specifi cations.

\section{Patterns for different software development activities}

Patterns are a means to reuse software development knowledge on different levels of abstraction. Patterns classify sets of software development problems or solutions that share the same structure.

Patterns have been introduced on the level of detailed object oriented design [GHJV95]. Today, patterns are defi ned for different activities. Problem Frames [Jac01] are patterns 

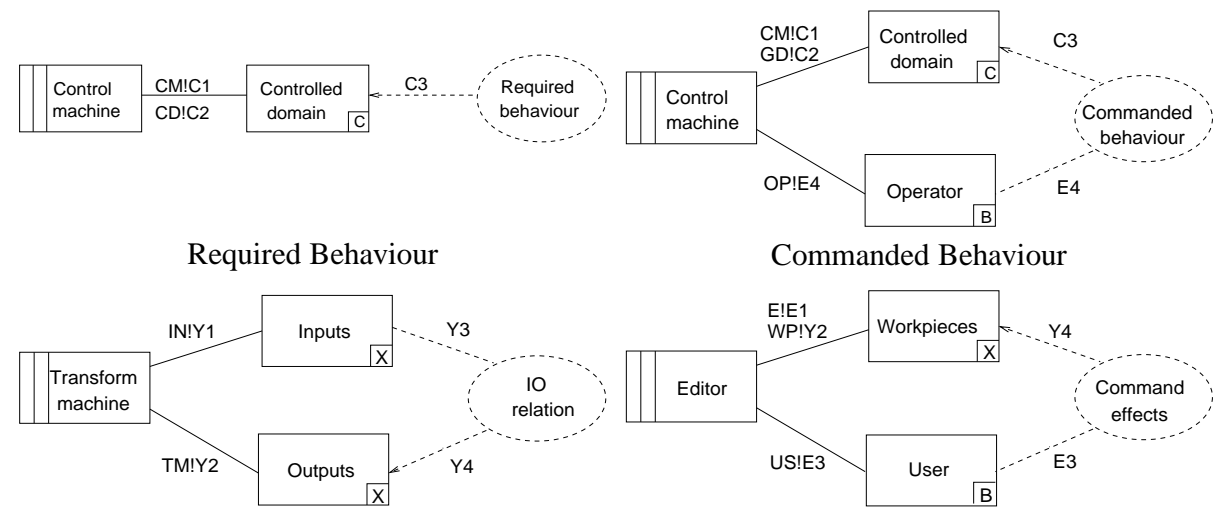

Commanded Behaviour

Transformation

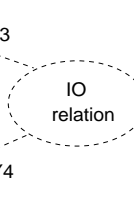

ation

Hitor

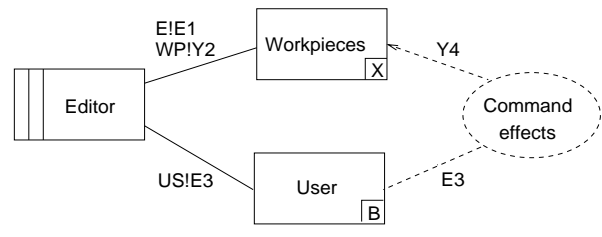

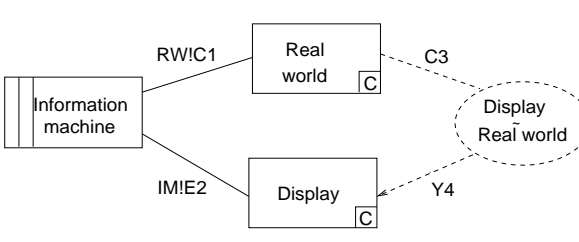

Information Display
Workpieces

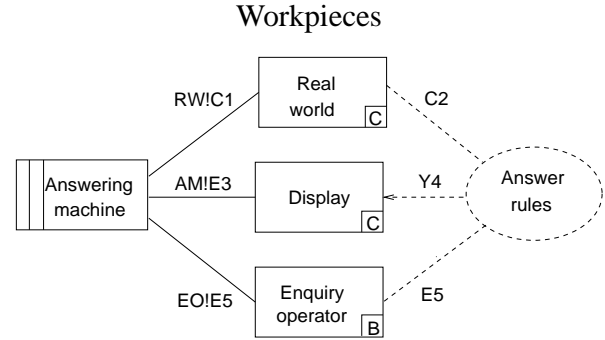

Commanded Information(Simple IS)

Fig. 3. Frame diagrams

that classify software development problems. Architectural styles are patterns that characterize software architectures [SG96,BCK98]. They are sometimes called "architectural patterns". Design Patterns are referred to as "micro-architectures", while frameworks are considered as less abstract, more specialized. Finally, idioms are low-level patterns related to specifi c programming languages $\left[\mathrm{BMR}^{+} 96\right]$, and are sometimes called "code patterns".

Using patterns, we can hope to construct software in a systematic way, making use of a body of accumulated knowledge, instead of starting from scratch each time. In the following, we briefly introduce problem frames and architectural styles, which will be used in our method.

\subsection{Problem Frames}

Jackson [Jac01] describes problem frames as follows:

A problem frame is a kind of pattern. It defi nes an intuitively identifi able problem class in terms of its context and the characteristics of its domains, interfaces and requirement.

For each problem frame, a frame diagram is set up (cf. Figure 3), which contains the different parts involved. Plain rectangles denote application domains. The characteristics of these domains play an important role in the application of a problem frame to a 
problem. A problem frame features also a machine domain denoted by a rectangle with a double vertical stripe, and a requirement denoted by a dashed oval. The connecting lines represent interfaces that consist of so-called "shared phenomena".

Jackson distinguishes causal domains that may control some shared phenomena (e.g. events) at the interface with another domain, biddable domains (people), and lexical domains that are physical representation of data. Causal phenomena (e.g. events) are caused or controlled by some domain, and can cause in turn other phenomena. Symbolic phenomena (e.g. values) can be changed, but cannot change themselves or cause changes elsewhere.

Jackson [Jac01] defi nes fi ve basic frames (that are variants of those given in [Jac95]). These (and a sixth derived problem frame) are briefly presented below. For each problem frame, we quote the description given by Jackson [Jac01] (see also Fig. 3).

Required Behaviour "There is some part of the physical world whose behaviour is to be controlled so that it satisfi es certain conditions. The problem is to build a machine that will impose that control." The " $\mathrm{C}$ " in the frame diagram indicates that the domain Controlled domain must be causal. The machine is always a causal domain (so an explicit " $\mathrm{C}$ " is not needed). The notation "CM!C1" means that the causal phenomena $C 1$ are controlled by the Control machine $C M$. The dashed line represents a requirements reference, and the arrow shows that it is a constraining reference.

Commanded Behaviour "There is some part of the physical world whose behaviour is to be controlled in accordance with commands issued by an operator. The problem is to build a machine that will accept the operator's commands and impose the control accordingly." The "B" indicates that the domain Operator is a biddable domain, and the phenomena $E 4$ are the operator commands.

Transformation "There are some computer-readable input fi les whose data must be transformed to give certain required output fi les. The output data must be in a particular format, and it must be derived from the input data according to certain rules. The problem is to build a machine that will produce the required outputs from the inputs." The "X" indicates that Inputs and Outputs are lexical (inert) domains.

Workpieces "A tool is needed to allow a user to create and edit a certain class of computer processable text or graphic objects, or similar structures, so that they can be subsequently copied, printed, analysed or used in other ways. The problem is to build a machine that can act as this tool."

Information Display "There is some part of the physical world whose states and behaviour is continually needed. The problem is to build a machine that will obtain this information from the world and present it at the required place in the required form." Here, the purpose of the machine is to display things that happen in the real world. Both domains are causal. $Y 4$ are symbolic requirement phenomena.

Commanded Information is derived from the Simple IS frame [Jac95]. There is some part of the physical world whose states and behavior are needed upon requests from an operator. The problem is to build a machine that will obtain this information from the world and present it at the required place in the required form.

Let us note that these problem frames do not cover every conceivable problem class. Some more problem frames have been identifi ed by Souquières and Heisel [SH00]. 


\subsection{Architectural Styles}

According to Bass, Clements, and Kazman [BCK98],

the software architecture of a program or computing system is the structure or structures of the system, which comprise software components, the externally visible properties of those components, and the relationships among them.

Architectural styles are patterns for software architectures. A style is characterized by [BCK98]:

- a set of component types (e.g., data repository, process, procedure) that perform some function at runtime,

- a topological layout of these components indicating their runtime interrelationships,

- a set of semantic constraints (for example, a data repository is not allowed to change the values stored in it),

- a set of connectors (e.g., subroutine call, remote procedure call, data streams, sockets) that mediate communication, coordination, or cooperation among components.

Important architectural styles are the following:

- Data-Centered with substyles Repository and Blackbord

- Data Flow with substyles Batch Sequential and Pipe-and-Filter

- Virtual Machine with substyles Interpreter and Rule-Based Systems

- Call-and-Return with substyles Main Program and Subroutine, Layered, ObjectOriented or Abstract Data Types

- Independent Components with substyles Communicating Processes and Event Systems (implicit/explicit invocation)

When choosing an architecture for a system, usually several architectural styles are possible, which means that all of them could be used to implement the functional requirements. Which architectural style is the most appropriate must then be decided using non-functional criteria such as effi ciency, scalability, or modifi ability. How such a choice is made is illustrated in Section 4.

\subsection{Design Patterns}

Design patterns [GHJV95] are used on a lower level of abstraction than problem frames or architectural styles. They provide concrete means to combine objects, or classes, respectively. In our overall software lifecyle, they would be used after an architectural style has been chosen. This step is beyond the scope of this paper.

\section{An agenda for pattern-based specification and design}

We now present our method for carrying out a pattern-based software lifecycle as shown in Figures 1 and 2. As a means of presentation, we use the agenda concept [Hei98]. An agenda is a list of steps or phases to be performed when carrying out some task in the 
Table 1. Agenda for pattern-based specification

\begin{tabular}{|c|c|c|c|}
\hline No. & Description & Result & Validation \\
\hline 1. & $\begin{array}{l}\text { Fit the problem into an ap- } \\
\text { propriate problem frame. }\end{array}$ & $\begin{array}{l}\text { Instantiated } \\
\text { frame dia- } \\
\text { gram }\end{array}$ & $\begin{array}{l}\text { All important issues of the problem must be } \\
\text { treated adequately, see also [Jac01]. }\end{array}$ \\
\hline 2. & $\begin{array}{l}\text { Set up a formal specifica- } \\
\text { tion for each domain of } \\
\text { the instantiated frame di- } \\
\text { agram (including the ma- } \\
\text { chine domain) and the re- } \\
\text { quirements. }\end{array}$ & $\begin{array}{l}\text { Set of formal } \\
\text { specifica- } \\
\text { tions }\end{array}$ & $\begin{array}{l}\text { - The specification must be coherent with } \\
\text { the instantiated problem frame diagram. } \\
\text { - The shared phenomena must belong to the } \\
\text { interfaces of all domains where they are } \\
\text { visible. } \\
\text { - Control of phenomena must be taken into } \\
\text { account. } \\
\text { - The specification } S \text { of the machine do- } \\
\text { main (in combination with the domain } \\
\text { knowledge } D \text { ) must suffice to satisfy the } \\
\text { requirements } R \text {, i.e., } S \wedge D \rightarrow R \text { must } \\
\text { hold. }\end{array}$ \\
\hline 3. & $\begin{array}{l}\text { Choose an appropriate ar- } \\
\text { chitectural style for struc- } \\
\text { turing the machine do- } \\
\text { main and instantiate it. }\end{array}$ & $\begin{array}{l}\text { Architectural } \\
\text { diagram and } \\
\text { informal text }\end{array}$ & $\begin{array}{l}\text { The chosen architecture must be able to satisfy } \\
\text { the machine specification. }\end{array}$ \\
\hline 4. & $\begin{array}{l}\text { Set up a formal specifica- } \\
\text { tion of all components ob- } \\
\text { tained in Step } 3 \text { and of the } \\
\text { overall system (i.e., spec- } \\
\text { ify how the components } \\
\text { cooperate). }\end{array}$ & $\begin{array}{l}\text { Set of formal } \\
\text { specifica- } \\
\text { tions }\end{array}$ & $\begin{array}{l}\text { - The formal specification must correspond } \\
\text { to the architectural diagram. } \\
\text { - The overall specification must be a refine- } \\
\text { ment of the machine specification devel- } \\
\text { oped in Step } 2 \text {. } \\
\text { - The constraints imposed by the chosen } \\
\text { architectural style must be satisfied. }\end{array}$ \\
\hline
\end{tabular}

context of software engineering. The result of the task will be a document expressed in some language. Agendas contain informal descriptions of the steps, which may depend on each other. Agendas are not only a means to guide software development activities. They also support quality assurance, because the steps may have validation conditions associated with them. These validation conditions state necessary semantic conditions that the developed artifact must fulfi 11 in order to serve its purpose properly.

Table 1 shows an agenda that precisely describes how to carry out and validate the fi rst steps of the lifecycle proposed in Figure 2. A precondition for the applicability of the agenda is that the problem is suffi ciently small that it may be fi tted into one problem frame. Complex problems have to be decomposed fi rst, for example by projection, as described by [Jac01].

Step 1 of the agenda is performed in principle as described by Jackson [Jac01]. To find the right problem frame, the structure of the frame diagram and the domain characteristics as described in Section 2.1 must be taken into account. However, this is not as straightforward as it might seem, because we fi rst need to choose between possibly different viewpoints on the problem. For instance, the choice of taking into 
Table 2. Problem frames and related architectural styles

\begin{tabular}{|l|l|}
\hline Problem Frame & Architectural Style \\
\hline \hline Required Behaviour & Communicating Processes, \\
Commanded Behaviour & Event/Action, Process Control \\
\hline Transformation & Repository, Batch Sequential, Pipe and Filter, \\
Workpieces & Virtual Machine, Layered, ADT/OO, Event Systems \\
\hline $\begin{array}{l}\text { Information Display } \\
\text { Commanded Information }\end{array}$ & $\begin{array}{l}\text { Repository, } \\
\text { Blackboard }\end{array}$ \\
\hline
\end{tabular}

account a user/operator influences the choice of problem frame, and it also changes the characteristics of the domains and phenomena. We think it is worthwhile to examine for each problem frame whether we fi nd a meaningful instantiation of it or else a clear reason why not.

Once the choice of a problem frame is made, we rely on the structure provided by the problem frame to proceed and establish a corresponding formal specification [CR00].

Step 2 uses the instantiated frame diagram from Step 1 that determines the structure of the formal specifi cation to be set up. For each box in the instantiated frame diagram, a specifi cation must be given. The validation condition "coherence of the instantiated frame diagram and specifi cation" means that the phenomena at the interfaces of the requirements box must be used in expressing the requirements. Moreover, the shared phenomena that are given in the instantiated problem frame must belong to the interfaces of the respective domain specifi cations. A domain which is in control of a shared phenomenon must be able to produce that phenomenon as an output, and a domain which is able to observe a phenomenon of which it is not in control must be able to take the phenomenon as an input. The domain knowledge $D$ mentioned in the last validation condition of Step 2 refers to the specifi cation of the application domains, i.e. the domains of the instantiated problem frame other than the machine domain.

Step 3 uses the specifi cation of the machine domain developed in Step 2. This specifi cation describes the machine to be developed, whose structure will be determined by the architectural style. Several possible architectural styles should be explored and assessed according to those non-functional criteria that are regarded to be important for the given problem.

Table 2 gives heuristics for performing Step 3. It has been developed from the general characteristics of the involved problem frames and architectural styles as well as by conducting several case studies. It shows rules of thumb giving hints which architectural styles to consider fi rst.

As can be seen, there are several architectural styles associated to each problem frame. Which one is fi nally chosen depends on non-functional requirements. It remains to make these explicit in order to really guide the transition from a problem frame to an architectural style. 


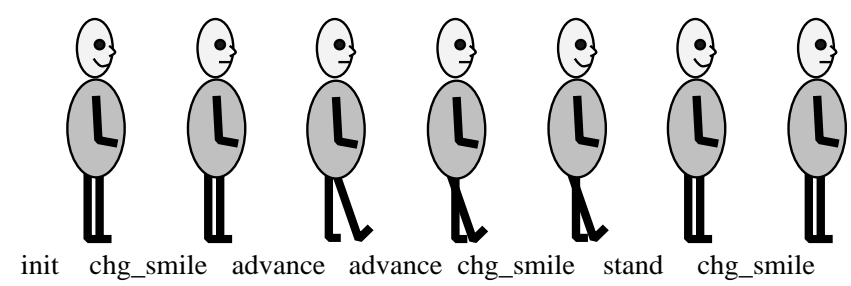

Fig. 4. The movements of the robot

For the problem frames Transformation and Workpieces, we have quite a number of architectural styles to consider. This is due to the fact that these problem frames cover most of the "classical" software development problems and that they are less constraining than the other frames. For Required Behaviour and Commanded Behaviour, we should consider architectural styles that are well suited for reactive systems, and for Information Display and Commanded Information, it seems natural to choose datacentered architectures.

Step 4 uses the architectural style instantiated in Step 3 to develop a specifi cation that formally describes the chosen architecture. That instantiation determines a set of components that structure the system to be developed. It also shows the cooperations between these components. For each component a formal specifi cation must be given. Furthermore, it must be specifi ed how the components cooperate. Such an architectural specification is the basis for detailed design and implementation. The most important validation condition associated with Step 4 of the agenda is to show that the chosen architecture indeed correctly implements the machine specifi ed in Step 2, i.e., that the architectural specification refi nes the machine specifi cation. Because we use formal specifi cations, this validation condition can be demonstrated in a rigorous or formal way.

In the following section, we demonstrate the application of the agenda by means of a concrete example.

\section{Case study: robot simulation}

This case study is taken from [HL97], where it was used to illustrate different architectural styles. Here, we demonstrate how the most suitable architectural style can be found in a systematic manner, performing the steps of the agenda presented in Table 1.

The task is to build a system simulating a simple robot. This robot can make the movements shown in Figure 4: it can advance by moving its right or its left leg; it can stand still; and it can smile or not. The robot can be modeled as an automaton with three states: standing, left_up and right_up as shown in Figure 5. To each state a boolean value is associated indicating whether the robot is smiling or not. The initial state is standing and smiling.

The robot is defi ned by the abstract data type ROBOT where the states are defi ned as constants and the movements as transitions from one state to another, except for smiling, 


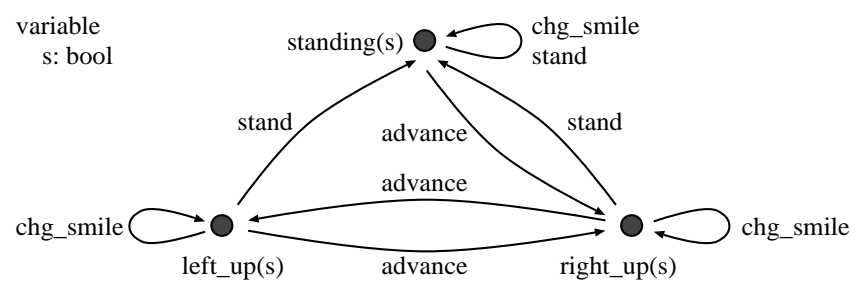

Fig. 5. The robot automaton

which is defi ned by a boolean value: true for smiling. For each state a predicate is defi ned deciding if the robot is in this state.

The input for the system to be built is a list of commands to be executed by the robot, i.e., a list consisting of the elements stand, advance and chg_smile. The output is a list of pairs, where the fi rst component of each pair is the current state of the robot, and the second component of each pair is the list of commands not yet executed. Each command must be executed, and the intermediate states entered during execution of the command list must be given as an output.

Step 1: Choice and Instantiation of a Problem Frame We consider the problem frames (cf. Figure 3) one by one and give reasons for each problem frame why it is rejected or accepted.

Required Behaviour The "C" says that the Controlled Domain must be causal. Since the robot is defi ned by an abstract data type, the domain corresponding to the robot is not causal but lexical. Moreover, the problem frame Required Behaviour does not let us distinguish between the input domain (being a list of commands) and the output domain (being a list of pairs). Hence, we reject this problem frame.

Commanded Behaviour This problem frame must be rejected for the same reasons as before. Moreover, we cannot fi nd a domain corresponding to the Operator domain.

Information Display Here, the purpose of the machine is to display things that happen in the real world. Both domains are causal, which does not fi t well with the robot problem.

Commanded Information This frame must be rejected, because we cannot fi nd an Enquiry operator and because the domains involved in the robot problem are not causal.

Workpieces This problem frame is more promising than the ones considered before, because we have a lexical domain here. The workpieces are the robot's state, together with the current command list. However, we cannot fi nd an instantiation for the User domain, because command lists are not biddable. Hence, we fi nally reject the Workpieces frame.

Transformation It is this frame that we fi nally choose for our problem. A lexical input list is transformed into a lexical output list. The relation between the two lists is given by the robot automaton. Figure 6 shows the instantiated frame diagram. 


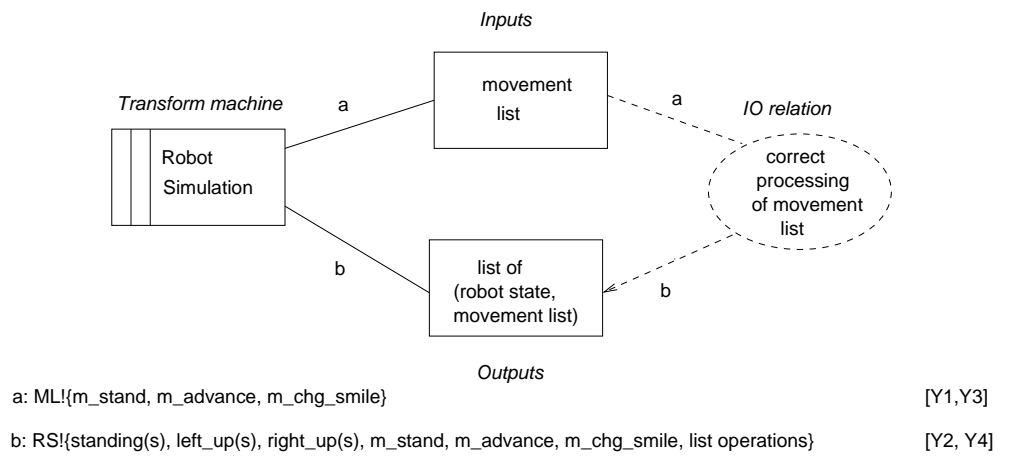

Fig. 6. The robot problem fitted into the Transformation problem frame

Step 2: Structured Requirements Specification Having chosen a problem frame for the robot problem, we must give a specifi cation of all the domains involved and of the requirements. As a specifi cation language, we use LOTOS [BB87], because LOTOS is one of the specifi cation languages allowing us to defi ne software architectures, and especially the interaction of different components, in a suitable way.

Specification of the Inputs Domain. As shown in Figure 6, the input domain is a list of movement commands.

The movements are defi ned by the type MVT with three constants m stand, m advance and m_chg_smile.

The robot will be asked to execute several movements collected in a list. This list is defi ned by an abstract data type M LIST whose defi nition is straightforward.

Specification of the Outputs Domain. The output consists of a list of pairs, whose fi rst element is the current state of the robot and whose second element is the list of movements yet to be performed.

The defi nition of the abstract data type ROBOT reflects exactly the automaton given in Figure 5.

To defi ne the Outputs domain O_ LIST, a data type VALUE must be defi ned as the Cartesian product (with constructor make) of the two types ROBOT and M_LIST. The type O_LIST of lists of elements of type VALUE is then defi ned in much the same way as the type M_LIST.

Specification of the $I O$ relation. The IO relation says that, given a list of commands, the robot simulation must execute that list of commands one by one and output the current state of the robot after execution of each command, together with the commands yet to be executed.

For example, if the input command list has the form $\left(m_{1}, m_{2}, m_{3}, \ldots m_{k}\right)$ then the output list has the form

$\left(\left(m_{1}(\right.\right.$ init of robot $\left.),\left(m_{2}, m_{3}, \ldots m_{k}\right)\right),\left(m_{2}\left(m_{1}(\right.\right.$ init of robot $\left.\left.)\right),\left(m_{3}, \ldots m_{k}\right)\right)$, $\ldots,\left(m_{k}\left(\ldots\left(m_{3}\left(m_{2}\left(m_{1}(\right.\right.\right.\right.\right.$ init of robot $\left.\left.\left.\left.)\right)\right)\right) \ldots\right)$, empty $\left.)\right)$

where $m(r)$ denotes the robot state that is reached from state $r$ by executing movement 
$m$. This requirement is defi ned by a predicate is_correct which takes a movement list and and output list as its arguments. This predicate is defi ned in a type IO_REL.

Specification of the Machine Domain Robot Simulation. For each input list, the robot simulation must produce an output list in such a way that the two lists are in the relation is_correct.

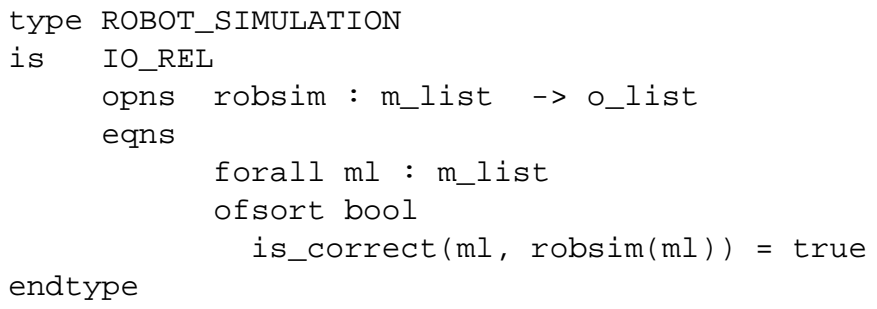

Steps 3 and 4: Architectural design of the robot We will explore several possibilities to structure the machine domain specifi ed in Step 2. The non-functional criteria for assessing the different architectures will be effi ciency and simplicity. Moreover, we give a specifi cation of the top-level behavior for each considered architecture. For reasons of space, we cannot give the specifi cations of the different components.

All architectures we will consider in the following have the same interface. This interface consists of an input channel START and an output channel OUTPUT, where START corresponds to interface $a$ and OUTPUT corresponds to interface $b$ of Figure 6 .

The list of movements to be processed is given in one step. The simulation must show the intermediate states of the robot when processing the input list. Hence, instead of producing the output list at once, the machine will produce the elements of the output list one by one. Then, the correctness condition required to be proven in Step 4 of the agenda is that the sequence of events occurring on gate OUTPUT is an output list that is in relation IO_rel with the input list.

The gate START is used to start the simulation, yielding in the following top-level behavior:

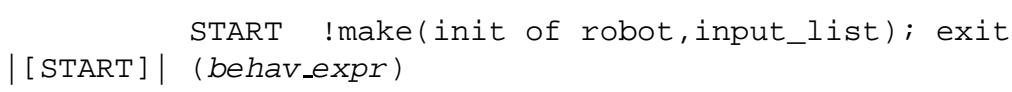

The different architectures will result in different defi nitions of behav_expr.

The Repository Architecture. The basic idea is to use a repository that contains the current state of the robot and the list of commands still to be executed. There are three components, one for each command. These components change the state according to the automaton and discard the fi rst element of the command list.

Figure 7 illustrates the repository architecture, where channel names R, W and RW denote the read, write and read/write access to the repository, respectively. The component Init_sm serves to write the initial state of the robot and the initial command list into the repository.

The components try to access the shared memory in parallel in order to execute the movement they are responsible for. Each of them fi rst reads the list of movements. If the first movement is the one it is responsible for, the movement is executed, the robot 


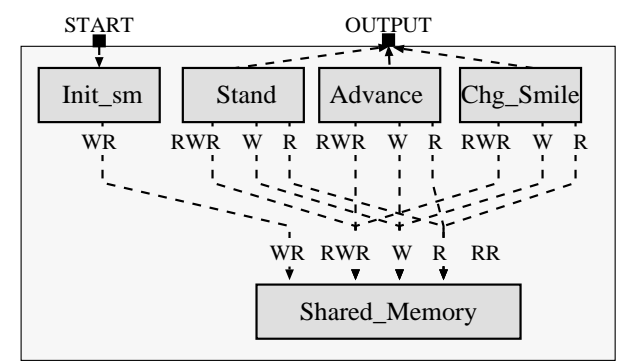

Fig. 7. The repository architecture for the robot

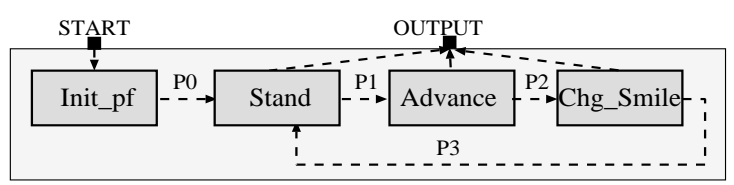

Fig. 8. The pipe/filter architecture of the robot

state changed, and the new state and the rest of the movement list is written back in the shared memory. If the movement cannot be executed by the component that has been granted access, it writes back the unchanged state in order to unlock the shared memory. The top-level behavior of this architecture is as follows:

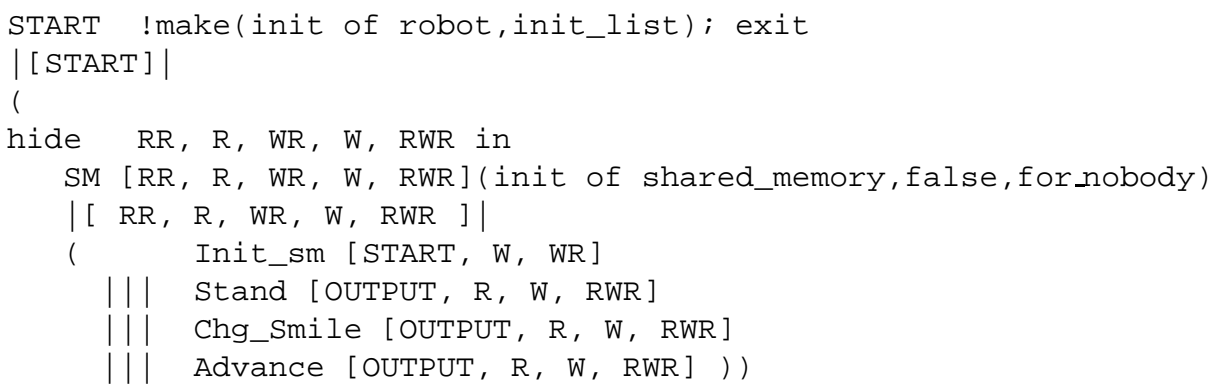

This architecture has the disadvantage that the system implementation must guarantee fairness, i.e. each component must be given the chance to access the shared memory. Otherwise, an infi nite number of unsuccessful accesses is possible, and the system does not terminate (live-lock).

The Pipe-and-Filter Architecture. In the pipe/fi lter modeling, we can make sure that each component is given the possibility to execute its movement if required. The idea is to have a line of fi lters. Each fi lter inspects the movement list. If it can execute the movement, it does so and hands the new robot state and the new movement list to the next fi lter. Otherwise, it passes on the unchanged data. Again, we need an initializing component, called here Init_pf. The architecture is shown in Figure 8. The top-level behavior of this architecture is as follows: 

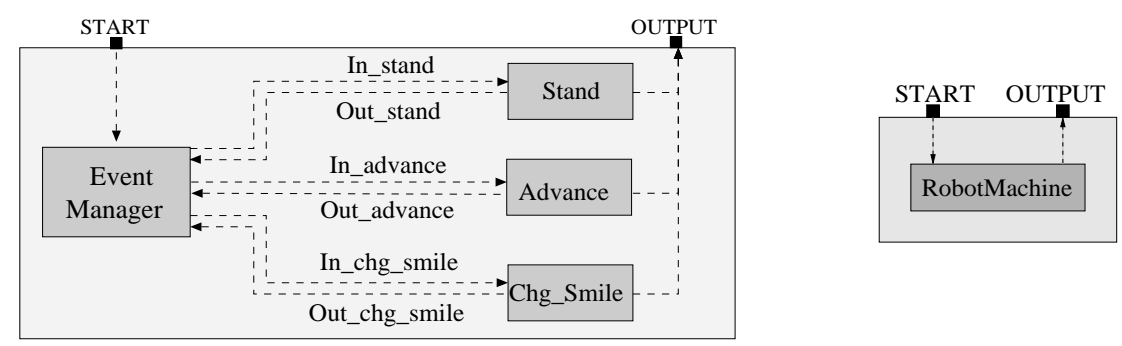

Fig. 9. The event system and the virtual machine architectures for the robot

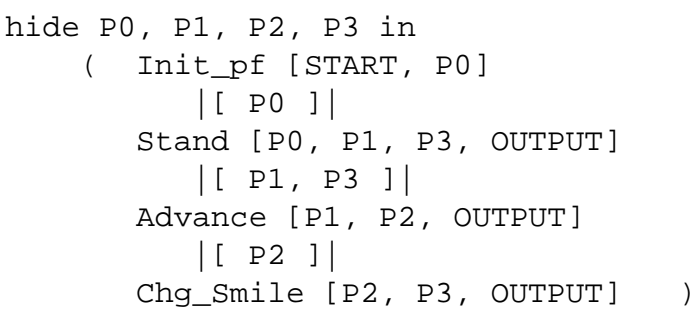

This solution is better than the repository architecture because it always terminates. It is not ideal, however, because each component must inspect the data, even if it cannot process them.

The Event System Architecture. The event system style can be used to overcome the disadvantages of the previous two architectures. An event manager inspects the movement list and passes the data only to the component that can process them. The initial state of the robot and the movement list are given to the event manager. No initialization component is required. This architecture is shown on the left-hand side of Figure 9. We have the following overall behavior:

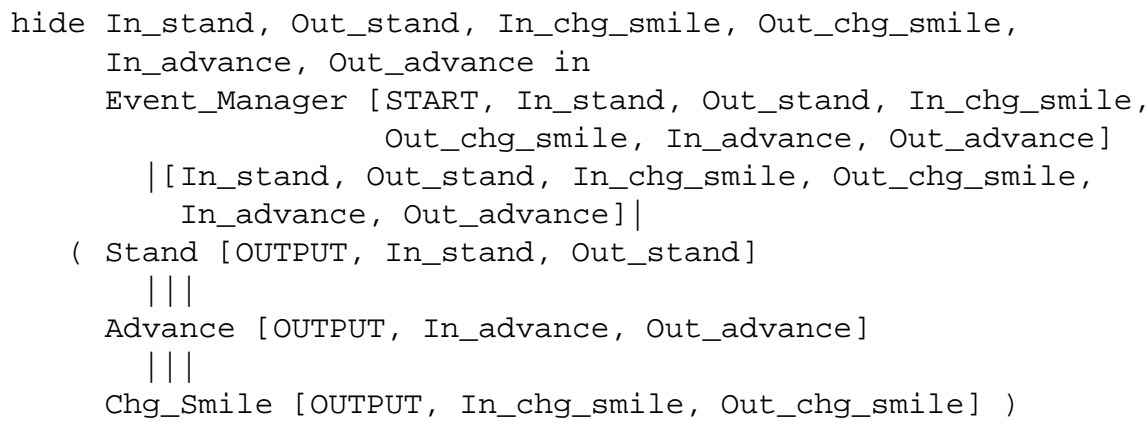

The components executing the movements are much simpler now than in the other architectures.

The Virtual Machine Architecture. The architecture can be improved once more. We should not have three components that can only execute a single command, but a virtual 
machine that can execute all three commands. This architectural style seems to be the most natural one, because virtual machines are well suited for simulation tasks. This architecture is shown on the right-hand side of Figure 9. It is quite simple:

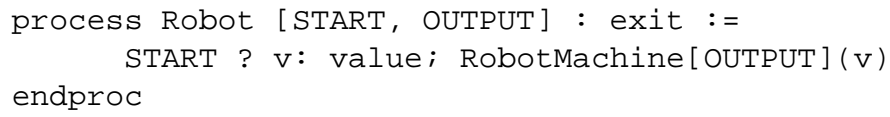

where the process RobotMachine just recursively processes the given movement list contained in $\mathrm{v}$.

This example shows that Table 2 can only give hints which architectural styles should be considered when developing an architecture for a given problem that was previously fi tted into some problem frame. We have demonstrated that several architectures yield correct implementations. However, some of them are better suited than others. The reasons for preferring one architecture over another were effi ciency as well as simplicity and elegance. For such a choice, no general rules can be given. However, the architectural styles provide us with an overall structure of the system to be developed. As we have shown, several such structures should be explored in search of the optimal one. The structure fi nally chosen is the starting point of the subsequent development steps.

For further validation of our approach, we have also carried out other case studies using CASL [CH03].

\section{Conclusions}

Methodological issues in writing specifi cations are many, and we would like to point to related work that addresses issues complementary to ours. Roggenbach and Mossakowski [RM02] address the writing of readable specifi cations in CASL, avoiding semantic pitfalls (these concerns are also addressed in the CASL reference manual [BM02]). Bidoit, Hennicker and Kurz [BHK02] explore the use of observability concepts which are found to be useful and relevant for writing specifi cations. Blanc [Bla02] proposes guidelines for the iterative and incremental development of specifi cations.

In this paper, we have introduced a methodology for formal specifi cation that is systematic and that stresses reuse of previously acquired knowledge. Both patterns and agendas are a means to represent knowledge. Patterns are abstractions of the products developed during the software lifecycle, and reuse is achieved by instantiating a pattern. Agendas, on the other hand, are explicit representations of process knowledge. Both concepts are orthogonal, and in order to base the software development process as much as possible on previously acquired knowledge, the two concepts should be used in combination. In particular, the contributions of this paper are:

- We have elaborated a software lifecycle where patterns play an important and welldefi ned role.

- We have developed an agenda that gives guidance how to perform this pattern-based software lifecycle in a systematic way.

- We have shown how to combine problem frames, architectural styles and formal specifi cations. So far, these three were considered in isolation; no explicit connection between them has previously been established. 
In the future we will provide methodological support also for the subsequent development steps of the software lifecycle proposed in Figure 2. In particular, this will involve the application of design patterns. Furthermore, we will investigate problem decomposition and multiframe problems in more detail.

Acknowledgments. We thank Thomas Santen, Carsten von Schwichow and an anonymous referee for their helpful comments on this paper.

\section{References}

[BB87] T. Bolognesi and E. Brinksma. Introduction to the ISO specification language LOTOS. Computer Networks and ISDN Systems, North-Holland, 14:25-59, 1987.

[BCK98] L. Bass, P. Clements, and R. Kazman. Software Architecture in Practice. AddisonWesley, 1998.

[BHK02] M. Bidoit, R. Hennicker, and A. Kurz. On the Integration of Observability and Reachability Concepts. In Proc. 5th Int. Conf. Foundations of Software Science and Computation Structures (FOSSACS'2002), LNCS 2303, pages 21-36. Springer Verlag, 2002.

[Bla02] B. Blanc. Prise en compte de principes architecturaux lors de la formalisation des besoins - Proposition d'une extension en CASL et d'un guide méthodologique associé. Thèse de Doctorat, ENS Cachan, 2002.

[BM02] M. Bidoit and P. Mosses. CASL User Manual, 2002. http: //www.brics.dk/Projects/CoFI/.

$\left[\mathrm{BMR}^{+} 96\right]$ F. Buschmann, R. Meunier, H. Rohnert, P. Sommerlad, and M. Stal. PatternOriented Software Architecture: A System of Patterns. John Wiley \& Sons, 1996.

[CH03] C. Choppy and M. Heisel. Systematic transition from problems to architectural designs. Technical report, Université Paris Nord, 2003. To appear.

[CR00] C. Choppy and G. Reggio. Using CASL to Specify the Requirements and the Design: A Problem Specific Approach. In Recent Trends in Algebraic Development Techniques, LNCS 1827, pages 104-123. Springer Verlag, 2000.

[GHJV95] E. Gamma, R. Helm, R. Johnson, and J. Vlissides. Design Patterns - Elements of Reusable Object-Oriented Software. Addison Wesley, Reading, 1995.

[Hei98] M. Heisel. Agendas - a concept to guide software development activites. In R. N. Horspool, editor, Proc. Systems Implementation 2000, pages 19-32. Chapman \& Hall London, 1998.

[HL97] M. Heisel and N. Lévy. Using LOTOS patterns to characterize architectural styles. In M. Bidoit and M. Dauchet, editors, Proceedings TAPSOFT'97, LNCS 1214, pages 818-832. Springer-Verlag, 1997.

[Jac95] M. Jackson. Software Requirements \& Specifications: a Lexicon of Practice, Principles and Prejudices. Addison-Wesley, 1995.

[Jac01] M. Jackson. Problem Frames. Analyzing and structuring software development problems. Addison-Wesley, 2001.

[RM02] M. Roggenbach and T. Mossakowski. What is a good CASL specification, 2002. WADT.

[SG96] M. Shaw and D. Garlan. Software Architecture. Perspectives on an Emerging Discipline. Prentice-Hall, 1996.

[SH00] J. Souquières and M. Heisel. Structuring the first steps of requirements elicitation. Technical Report A00-R-123, LORIA, Nancy, France, 2000. 